\title{
RESISTENCIA BACTERIANA \\ EN INFECCIONES HOSPITALARIAS Y ADQUIRIDAS Y SU RELACIÓN CON HÁBITOS DE PRESCRIPCIÓN DE ANTIBIÓTICOS
}

\author{
Autores: \\ Jaime Silva-Cevallos' \\ Alexandra Montalvo $\mathrm{T}^{2}$ \\ Ruth Martínez ${ }^{2}$ \\ Rosa Palma² \\ Andrés Delgado'
}

Facultad de Ciencias de la Salud Eugenio Espejo. Departamento de Ciencias Básicas.

Universidad Tecnológica Equinoccial, Av. Occidental y Av. Mariana de Jesús, Apartado 17-01-2764, Quito - Ecuador

jsilva@ute.edu.ec.

A. Montalvo, R. Martínez, R. Palma

Hospital Regional Docente Pablo Arturo Suárez. Laboratorio de Microbiología Quito. Ecuador. 



\section{Resumen}

La resistencia bacteriana está en relación con el uso, mal uso y abuso de los antibióticos, es un problema global, complejo; incluye un gran número de especies bacterianas de importancia médica y es de difícil control por su multicausalidad. Uno de los factores más importantes está relacionado con la prescripción empírica de los antibióticos. El objetivo de la investigación es determinar el soporte en el que se sustenta la prescripción de antibióticos, conocer los hábitos de prescripción empírica relacionarlos con la prevalencia y perfil de resistencia de las bacterias aisladas en la comunidad y en el hospital. Se trata de un estudio descriptivo realizado en un hospital regional de tercer nivel en la ciudad de Quito.

Los antibióticos más prescritos empíricamente son los betalactámicos con o sin inhibidores de beta lactamasas 34 , cefalosporinas $30 \%$ y flluoroquinolonas 9 \%. Las bacterias más frecuentemente aisladas fueron escherichia coli 47 \% en la comunidad y 74 \% en el hospital, seguidos por otros gram negativos como enterobacter sp, proteus sp y staphylococcus aureus $5 \%$ y $13 \%$ respectivamente. Los perfiles de resistencia de gram negativos mostraron una alta resistencia para Ampicilina $67 \%$ a 88 \% y media para betalactámicos con inhibidores de beta lactamasas betalactámicos entre 19 y 33 \%,alta resistencia para cefalosporinas de 67 \% a 100 \%, así como a fluoroquinolonas 43 a 67 \%. En gram positivos la resistencia de staphylococcus aureus fue para oxacilina 33\%, eritromicina 47 \% norfloxacina $67 \%$ y ampicilina $100 \%$. Antibióticos con perfiles bajos de resistencia fueron amikacina, fosfomicina, nitrofurantoína y se registró resistencia para carbapenems; linezolid, clindamicina y vancomicina mantienen porcentajes de sensibilidad altos.

Los hábitos de prescripción empírica sobre el uso de antibióticos se relacionan con los perfiles de resistencia a las bacterias encontradas en un hospital o en el ambiente en general. La información contenida en este trabajo puede ayudar a desarrollar guías de tratamiento empírico y políticas de contención de la resistencia bacteriana.

Palabras clave: resistencia a antimicrobianos (AMR), agente antimicrobiano (AAM), hábito de prescripción

\section{Abstract}

Bacterial resistance is related to the use, misuse and abuse of antibiotics is a global problem, complex and includes a number of medically important bacterial species and is difficult to control for its multiple causes. One of the most important factors is related to the empirical prescription of antibiotics. The objective of the research is to determine the base of which is based antibiotic prescribing, prescribing habits know empirically relate the prevalence and resistance profile of bacteria isolated in the community and in the hospital. This is a descriptive study in a tertiary regional hospital in the city of Quito.

Keywords: antimicrobial resistance (AMR), antimicrobial (AAM), prescription habit 


\section{Introducción}

El problema de la resistencia a los antibióticos es global, complejo, incluye un gran número de especies bacterianas de importancia médica y es de difícil control por su multicausalidad (1).

La resistencia bacteriana está en relación con el uso, mal uso y abuso de los antibióticos, se considera que hasta un $50 \%$ de los antibióticos son usados de manera inadecuada para tratar infecciones virales y otros problemas que no pueden ser curados con antibióticos (2). Entre los factores asociados al paciente están automedicación, acceso libre a los antibióticos sin prescripción médica, falta de cumplimiento de dosis y tiempo de medicación; pero también existen otros relacionados al médico como prescripción inadecuada y sobreprescripción, existe evidencia sobre la relación de uso de antibióticos y resistencia bacteriana (3), sin mencionar la utilización de antibióticos en la industria alimenticia y agropecuaria que modifican la microbiota humana y contribuyen por presión selectiva al aparecimiento de bacterias resistentes. $(3,4)$

La resistencia bacteriana a los antimicrobianos $A M R$ fue declarada por la Organización Mundial de la Salud (OMS) como un problema de salud pública (5). Un gran número de países en Latinoamérica han implementado redes de vigilancia de la resistencia bacteriana y se han declarado alertas en todos los continentes $(6,7)$; sin embargo la información obtenida de varios centros asistenciales o diferentes áreas geográficas tiene un valor limitado por lo que es necesario realizar estudios locales en hospitales y comunidades (7). En los países en vías de desarrollo hay limitada información sobre prescripción y uso de antibióticos y su relación con emergencia de bacterias resistentes a los nuevos antimicrobianos, por tanto se conoce poco sobre los patrones de resistencia de las bacterias a los antibióticos y menos aún sobre políticas para reducir su consumo, a pesar del impacto económico que representa para los usuarios que los pagan, hospitales ó pacientes (8). Existe una emergencia global de resistencia a los antibióticos frecuentemente prescritos, la prescripción empírica debería ser guiada por patrones locales de sensibilidad a los antimicrobianos (9). México fue el primer país el Latinoamérica en tomar la decisión de legislar y no vender antibióticos sin prescripción médica luego de demostrar que era el país con la más alta tasa de resistencia bacteriana (10).

La Red de Vigilancia de la Resistencia Bacteriana en el Ecuador (Rednarbec) ha generado información sobre resistencia de bacterias centinela, desafortunadamente no difundida entre la comunidad médica. En el año 2007 en 18 hospitales y clínicas participantes, se reporta una resistencia acumulada en escherichia coli de 59 al $68 \%$ para sulfa-trimetoprim, de 72 al 82 \% para ampicilina y de 41 al $59 \%$ para ciprofloxacina dependiendo si fue aislada en muestras de hospital o de la comunidad (11). Estos antibióticos están entre los más vendidos en el país contribuyendo a la magnitud del problema (13).

La elección de un tratamiento empírico con agentes antimicrobianos AAM está determinada por muchos factores que van desde la formación académica, experiencia, guías de tratamiento, hasta la promoción e incentivos de la industria farmaceútica(7). La tendencia es a utilizar más frecuentemente antibióticos de amplio espectro, especialmente entre los médicos del área urbana y en algunos casos a usar los antibióticos de nueva generación favoreciendo la emergencia de bacterias resistentes en la comunidad(14). En el hospital las infecciones son producidas por bacterias que han desarrollado resistencia y requieren vigilancia: enterococcus faecium, staphylococcus aureus, klebsiella pneumoniae, acinetobacter baumanni, pseudomonas aeruginosa y enterobacter species, (7). Además existe preocupación porque ha declinado la investigación y desarrollo de nuevos antibióticos con el riesgo de no tener a futuro disponibilidad de AAM efectivos para el cuidado médico (14).

Este estudio tiene como objetivo establecer la frecuencia de aislamientos bacterianos en las muestras de origen comunitario COM y hospitalario HOS así como los patrones de resistencia de las bacterias a los antibióticos y relacionarlos con los hábitos de prescripción 
empírica de antibióticos de los médicos del Hospital Docente Pablo Arturo Suárez (HPAS) de la ciudad de Quito. También se busca evidenciar el grado de conocimiento y el estado de situación actual del problema de resistencia bacteriana en la población atendida por éste hospital, para contribuir a consolidar un sistema permanente de vigilancia y contención, que permita en el futuro identificar cambios tempranos en los patrones de resistencia bacteriana a los antibióticos o identificar la emergencia de cepas bacterianas multiresistentes.

\section{Metodología}

\section{Hábitos de prescripción}

Se aplicó una encuesta al 68\% de los médicos registrados del HPAS cuyo objetivo era determinar: (a) las fuentes de información y soporte para la prescripción de antibióticos, (b) conocer los hábitos de prescripción empírica de antibióticos, (c) investigar el grado de conocimiento sobre aspectos de vigilancia y estudios de resistencia bacteriana y (d) establecer si existía relación entre la elección de prescripción antibiótica empírica y los perfiles de resistencia de las bacterias aisladas en el laboratorio. Para determinar el uso de antibióticos se analizaron las preferencias de los médicos para utilizar antibióticos en forma general y por tipo de infección de acuerdo al hábito empírico registrado por el médico prescriptor.

Se realizaron entrevistas con los médicos de todos los servicios y especialidades, medicina general y medicina interna 16 $\%$, traumatología y ortopedia $18 \%$, cirugía general $15 \%$, ginecología y obstericia $10 \%$, pediatría $7 \%$, unidad de cuidados intensivos (UCI) $7 \%$ y emergencia $2 \%$, es decir las especialidades principales del HPAS. Los médicos no prescriptores fueron del área administrativa y anestesia.

\section{Prevalencia de microorganismos y Patrones de resistencia}

Se revisó un total de 1616 resultados positivos de cultivos y antibiogramas realizados en el Laboratorio de Microbiología del Hospital Docente Pablo Arturo Suárez, entre enero y diciembre del 2010. Los datos fueron ingresados al programa de vigilancia gratuito Whonet 5.6 (OMS, http://www.whonet.org) para su análisis. El programa fue actualizado con los cambios en la selección de antibióticos e interpretación de resultados recomendados para el $2010(15,16)$

Para el análisis se seleccionó sólo una cepa por especie por paciente- la primera en caso tuviera varios aislados- diferenciadas por procedencia y tipo de muestra. Se distribuyeron en dos grupos: aislados de origen comunitario (COM) los de muestras de pacientes ambulatorios provenientes de consulta externa y emergencia y aislados de origen hospitalario HOS los de pacientes internados en los diferentes servicios y de UCl.

Del análisis de 1498 cepas seleccionadas, la mayoría identificadas por especie, se obtuvieron el número y porcentaje de aislamientos para determinar la prevalencia de las diferentes especies bacterianas en la comunidad y en el hospital. Se realizó el análisis de los aislados más frecuentes según tipo de muestra y origen para determinar la frecuencia de microorganismos en muestras de orinas, respiratorias, de piel, tejidos blandos, hueso y de sitios estériles. Para las cepas aisladas se calculó el porcentaje de resistencia frente a cada agente antimicrobiano probado, usando el experto del programa Whonet 5.6.

\section{Evaluación de desempeño y calidad.}

Durante el estudio se entregó al Laboratorio de Microbiología del HPAS cuatro cepas ATCC codificadas con letras A (27853 pseudomona aeruginosa), B (51299 enterococo fecalis resistente a Vancomicina), C (25923 estafilococo aureus) y D (25922 eschericia coli) para su identificación y antibiograma, con el fin de evaluar el desempeño y calidad de los resultados que se estaban reportando. Además se revisaron los manuales de procedimientos para verificar los 
estándares de trabajo. Los exámenes microbiológicos fueron realizados siguiendo las normas CLSI y las recomendaciones de la Rednarbec a la cual pertenecía el Hospital desde el año 2007. La identificación se realizó con métodos manuales y galerías API para determinar la susceptibilidad de los microorganismos a los antibióticos y se utilizó el método de difusión en agar (técnica de Kirby Bauer). El laboratorio no realizó pruebas de concentración inhibitoria mínima (CIM).

\section{Resultados}

\section{Hábitos de prescripción}

La mayoría de médicos encuestados (71\%) desconocían que el Hospital pertenecía a una red de vigilancia de la resistencia bacteriana. Adicionalmente sólo $6 \%$ dijeron conocer los resultados sobre resistencia bacteriana del HPAS, 39 \% resultados locales o nacionales, 18 \% en Latinoamérica, 23 \% otros estudios y un $20 \%$ no contestó.

El hábito de prescripción empírica está influenciado entre otros factores por la experiencia, el uso de guías, protocolos, medicina basada en evidencias, experiencia y hasta cierto punto la costumbre y en otros casos el nivel de conocimiento sobre los patrones de resistencia en estudios realizados (22). En la prescripción de los antibióticos prima la experiencia y el criterio de los médicos, las guías hospitalarias no revierten mayor importancia. Diferentes fuentes sustentan la prescripción de antibióticos, la mayoría de médicos usan libros texto, guías de práctica, pero estudios demuestran que hasta un 80 \% hace uso extenso de la información de las compañías farmacéuticas (30).

La formación académica necesita ser más práctica y enseñar de forma fácil sobre la aplicación de antibióticos en situaciones reales y las guías hospitalarias deberían ser más amigables y promocionadas (29).

En este estudio, el $56 \%$ de los médicos respondieron seguir guías o protocolos para el uso de antibióticos en los servicios ó en el Hospital, sin embargo al preguntar sobre las guías utilizadas, ninguno de los encuestados mencionó guías propias del servicio o del hospital, un 29 \% no respondió, un 13 \% usa el Sanford, 15 \% refieren guías de especialidad de sociedades científicas internacionales y 4 \% usa guías del MSP para el uso de medicamentos básicos.

Un $68 \%$ de los encuestados dicen no tener restricciones para el uso de antibióticos en los servicios del HPAS. Quienes respondieron afirmativamente no diferencian entre lo que es restricción de uso para disminuir la resistencia bacteriana y limitaciones farmacológicas del uso de medicamentos ó limitaciones logísticas y de disponibilidad institucional.

En el sector público se promueve la sobreutilización de antibióticos (23), además se evidenció que no existe regulación en el uso y mal uso de los antibióticos, se manejan diagnósticos inciertos, priman las expectativas de los pacientes y consideraciones económicas.

Los médicos encuestados soportan las fuentes de información y los criterios para recetar antibióticos. En el resultado del antibiograma, el 47 \% basó su criterio en la formación académica, el 42 \% en las guías de tratamiento internacionales, el 42 \%, lo sustenta en las guías de tratamiento locales, literatura médica indexada y MBE y el Cuadro de medicamentos básicos del MSP. La experiencia aporta un $12 \%$, el cuadro de saldos de farmacia un $7 \%$ y la información de las empresas farmaceúticas presentadas en el vademécum un $6 \%$.

Los antibióticos más prescritos en la mayor parte de estudios son las penicilinas de amplio espectro con inhibidores de betalactamasas (28). En este estudio según las familias de antibióticos las penicilinas (16 \%) solas o con inhibidores de batalactamasas (22\%) son los más prescritos seguidos de cefalosporinas $31 \%$ y las quinolonas $11 \%$.

En orden de importancia, los medicamentos más prescritos por los médicos son: Ampicilina +IBL 18 \%, Cefazolina 12\%, Ciprofloxacina 
$9 \%$, Ampicilina $9 \%$, Amoxicilina 7\%, Cefalexina9\%, Ceftriaxona $9 \%$, estos a su vez se relacionan con los 50 medicamentos más vendidos en el Ecuador en el año 2010. Diez son antibióticos (ciprofloxacina, amoxicilina, amoxicilina+acido clavulánico, cefuroxima axetilo, azitromicina, sulfametoxazol+ trimetoprin, ampicilina, claritromicina, cotrimoxazol y cefadroxil).

A pesar de las dificultades para obtener información sobre los volúmenes comercialización de antibióticos, un estudio realizado en el 2004 reporta que el consumo global de antibióticos el país fue de 17,65 DDD/100o habitantes por día (Dosis Diarias Definidas), comparable a los de países desarrollados. Los subgrupos terapéuticos de mayor consumo fueron las penicilinas de amplio espectro y penicilinas asociadas a inhibidores de betalactamasas, que representaron más del $51 \%$ de la prescripción total de antibióticos $(20,24)$. El aumento en la prescripción de antibióticos hace necesario revisar los hábitos de prescripción de los médicos. Una forma de reducir las infecciones por microorganismos resistentes a los AAM es reducir la presión selectiva de los antibióticos disminuyendo su uso inapropiado en el hospital y la comunidad (26).

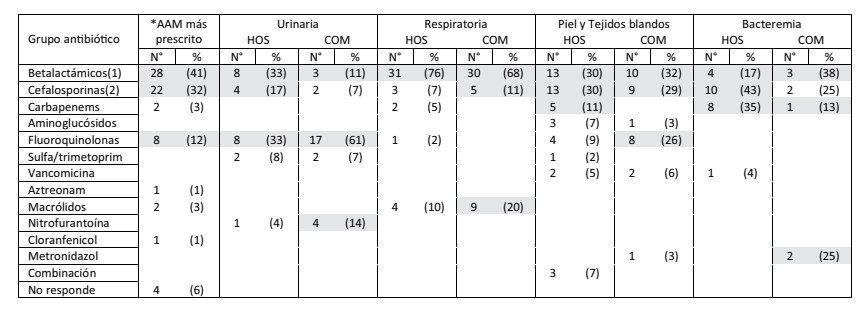

*Médicos que prescriben antibióticos en el HPAS. N= 68

(1) Betalactámicos solos o con inhibidores de batalactamasas

(2) Cefalosporinas de primera,segunda y tercera generación

Tabla 1. Hábitos de prescripción: antibióticos de elección empírica HPAS. 2010

\section{Prevalencia de microorganismos}

Los resultados de aislamientos bacterianos se muestran en la tabla (Tabla 1), la bacteria más aislada tanto en muestras hospitalarias como delacomunidadfueescherichia colicon $44 \%$ y $74 \%$ respectivamente existiendo una diferencia significativa, sin embargo la frecuencia en aislados urinarios fue muy similar $84 \%$ y $82 \%$. Estafilococo aureus fue la segunda bacteria más aislada con $13 \%$ y $5 \%$ respectivamente existiendo una diferencia significativa pero su mayor frecuencia se observó en los aislados de piel, tejidos blandos y hueso con $25 \%$ y 42 \% y sitios estériles con $19 \%$ y $33 \%$ respectivamente. (Tablas 2 y 3 ).

\begin{tabular}{|c|c|c|c|c|c|c|}
\hline Microorganismos N= 1489 & $\mathrm{~N}^{\circ}$ & \multicolumn{2}{|c|}{ HOS } & \multicolumn{2}{|c|}{ COM } & $p>0.05$ \\
\hline \multicolumn{2}{|l|}{ GRAM NEGATIVOS } & $\mathrm{N}^{\circ}$ & $\%$ & $\mathrm{~N}^{\circ}$ & $\%$ & \multirow[b]{2}{*}{$p>0.05$} \\
\hline Escherichia coli & 853 & \multirow{2}{*}{$\begin{array}{l}391 \\
51\end{array}$} & \multirow{2}{*}{$\begin{array}{l}-44 \\
-6\end{array}$} & \multirow{2}{*}{$\begin{array}{c}462 \\
7\end{array}$} & -74 & \\
\hline Pseudomonas aeruginosa & 58 & & & & -1 & $p>0.05$ \\
\hline Enterobacter sp. & 164 & \multirow{2}{*}{$\begin{array}{c}122 \\
61\end{array}$} & \multirow{2}{*}{$\begin{array}{l}-14 \\
-7\end{array}$} & 42 & -6 & $p>0.05$ \\
\hline Proteus sp. & 85 & & & 24 & -4 & \\
\hline Citrobacter sp. & 29 & \multirow{2}{*}{$\begin{array}{l}20 \\
20\end{array}$} & $\begin{array}{l}-7 \\
-2\end{array}$ & 9 & -1 & \\
\hline Klebsiella pneumoniae & 29 & & -2 & 9 & -1 & \\
\hline Otras enterobacterias & ${ }^{14}$ & \multirow[t]{2}{*}{6} & \multirow[t]{2}{*}{-2} & \multirow[t]{2}{*}{8} & \multirow[t]{2}{*}{-1} & \\
\hline \multicolumn{2}{|l|}{ GRAM POSITIVOS } & & & & & \\
\hline Enterococcus faecalis-faecium & 51 & \multirow{3}{*}{$\begin{array}{c}37 \\
113 \\
41\end{array}$} & \multirow{2}{*}{$\begin{array}{l}-4 \\
-13\end{array}$} & 14 & -2 & \\
\hline Staphylococcus aureus & 145 & & & 32 & -5 & $p>0.05$ \\
\hline Staphylococcus coagulasa neg & 58 & & -5 & 17 & -3 & \\
\hline Otros gram positivos & 8 & 4 & -1 & 4 & -1 & \\
\hline
\end{tabular}

Tabla 2. Aislamientos bacterianos. Hospital Pablo Arturo Suárez. Quito. Enero a Diciembre 2010

Otras enterobacterias como enterobacter sp. $14 \%$ y $6 \%$, proteus sp. 7 y $4 \%$, citrobacter sp, klebsiella pneumoniae y otras enterobacterias $2 \%$ y $1 \%$ respectivamente se aislaron indistintamente según su origen: urinario, respiratorio, piel, tejidos bandos y hueso ó sitios estériles. Entre los gram positivos estrepcococcus faecalis y faecium y estafilococus coagulasa negativos fueron aislados principalmente de vías urinarias y piel y tejidos blandos, así como staphylococcus coagulasa negativo que fueron más frecuentes en muestras de sitios estériles 23 y $22 \%$ y piel y tejidos blandos 3 y $6 \%$ respectivamente.

En general la prevalencia de microorganismos aislados corresponde a lo esperado en infecciones en otros estudios, sin embargo llama la atención la ausencia de aislamientos respiratorios de streptoccocus pneumoniae, haemophilus influenza y moraxella catharralis, los patógenos más frecuentes en infecciones respiratorias y podría asociarse con problemas de automedicación, tratamiento precoz con antibióticos o dificultades técnicas para su aislamiento (21). 


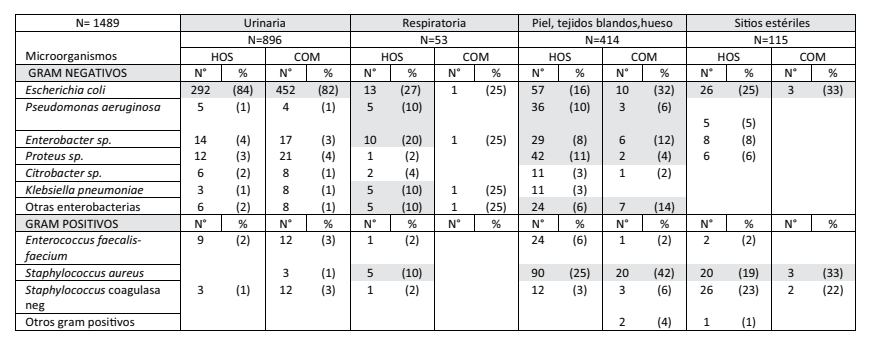

Tabla 3. Aislamientos de muestras de origen comunitario COM y hospitalario HOS . Hospital Pablo Arturo Suárez. Enero a Diciembre 2010

\section{Perfiles de resistencia}

El análisis de los perfiles de resistencia se hizo de las especies bacterianas más aisladas que representan el $83 \%$ de todos los aislados analizados y que son sugeridas para vigilancia por la OPS y los resultados se observan en las Tablas 4 y 5 .

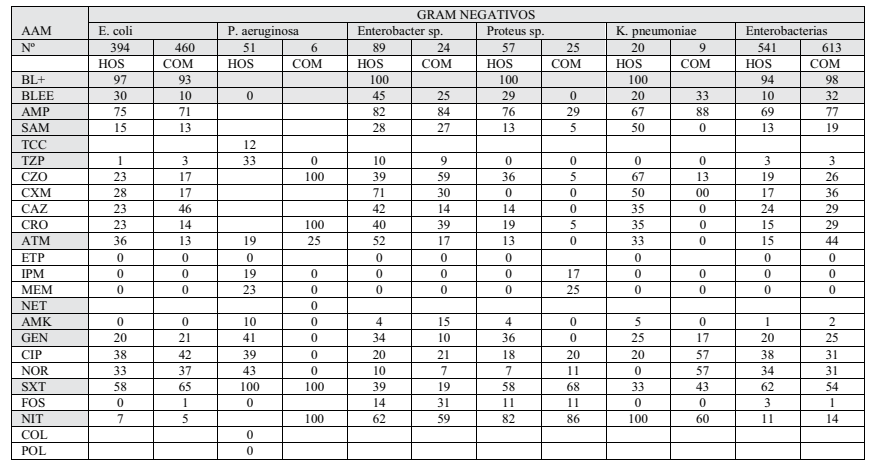

AMP: Ampicilina. AMK: Amikacina. CAZ: Ceftazidima. CLI Clindamicina. COL Colistin. CRO Ceftriaxona. CXM: Cefotaxima. CTX Cefotaxima. CZO Cefazolina. ERY: Eritromicina.ETP Ertapenem AZM Azitromicina. CIP: Ciprofloxacina.FOS Fosfomicina. FOX Cefoxitina. IPM: Imipenam. LNZ Linezolid.LVX Levofloxacina. MEM: Meropenem. NOR Norfloxacina.

ATM: Aztreonam. GEN: Gentamicina. GEH: Gentamicina alta potencia. POL Polimixi-

na. RIF Rifampicina. SAM: Ampicilina-Sulbactam. STH: Estreptomicina alta potencia.

TEH Tetraciclina alta potencia. VAN: Vancomicina. TCC Ticarcilina-Ac. Clavulánico. TZP:

Piperacilina-Tazobactam.. NET: Netilmicina. NIT Nitrofurantoína. SXT: Trimetoprim-Sulfametoxazol. PEN: Penicilina. OXA: Oxacilina.

Tabla 4. Resistencia bacteriana por bacterias de vigilancia y grupo antimicrobiano GRAM NEGATIVOS

\section{Gram Negativos}

En relación a las bacterias gram negativas aisladas, la ampicilina mostró alta resistencia de $67 \%$ al 88 \% tanto en la comunidad como en el Hospital, no así para los betalactámicos con inhibidores de betalactamasas cuya sensibilidad se mantiene sobre el $81 \%$ con excepción de HOS pseudomona aeruginosa con resistencia del 33 \%, enterobacter aislados COM y $\operatorname{HOS} 27 \%$ y $28 \%$ y klebsiella neumoniae HOS $50 \%$. (Tabla 4)

Una sensibilidad variable se observó en gram negativos con relación a cefalosporinas con tendencia a alta resistencia especialmente con pseudomona aeruginosa 100\%, enterobacter sp. $71 \%$ y klebsiella pneumoniae $67 \%$. Una resistencia promedio de $44 \%$ se observó para el Aztreonan con alta resistencia de enterobacter HOS del $52 \%$.

Los resultados muestran una alta sensibilidad para los carbapenems con excepción de pseudomona aeruginosa HOS y proteus sp. COM que muestran una resistencia de hasta un $25 \%$.

Los aminoglucósidos, especialmente amikacina muestran una alta sensibilidad a todos los aislados, no así gentamicina que muestra resistencia de $41 \%$ para seudomona aeruginosa $34 \%$ para enterobacter y $36 \%$ para proteus aislados del Hospital.

Las quinolonas muestran una sensibilidad disminuída de 57 a 67 \% para los aislados de pseudomona aeruginosa, escherichia coli y menor klebsiella pneumoniae HOS del 43 \%, manteniendo resistencias bajas para enterobacter y proteus sp.

La resistencia a sulfa trimetoprim para enterobacterias promedio del 54 \% contrasta con la sensibilidad alta de fosfomicina para enterobacterias. Nitrofurantoína mostró alta sensibilidad para escherichia coli menor a 7 \% sin embargo la resistencia es alta para otras enterobacterias y pseudomona entre $59 \%$ y $100 \%$.

\section{Gram positivos}

La alta resistencia para penicilina se observa para los aislados de staphylococcus aureus 96\%, enterococcus $34 \%$ y staphylococcus coagulasa negativos $94 \%$. (Tabla 5) 


\begin{tabular}{|l|c|c|c|c|c|c|}
\hline \multirow{3}{*}{ AAM } & \multicolumn{7}{|c|}{ GRAM POSITIVOS } \\
\cline { 2 - 7 } & \multicolumn{2}{|c|}{ S. aureus } & \multicolumn{2}{l}{$\begin{array}{l}\text { E. faecalis } \\
\text { E. faecium }\end{array}$} & \multicolumn{2}{l}{$\begin{array}{l}\text { Staph. coagulasa } \\
\text { neg }\end{array}$} \\
\hline N $^{\text {r }}$ & 113 & 32 & 37 & 14 & 41 & 17 \\
\cline { 2 - 7 } & HOS & COM & HOS & COM & HOS & COM \\
\hline PEN & 91 & 96 & 34 & 7 & 97 & 75 \\
\hline AMP & & 100 & & & & \\
\hline CTX & & & & & 100 & \\
\hline FOX & 28 & 31 & & & 61 & 20 \\
\hline AMK & & & & & & \\
\hline GEH & 11 & 12 & 15 & 17 & 17 & 0 \\
\hline CIP & 8 & 10 & 0 & 0 & 19 & 22 \\
\hline NOR & & 67 & 57 & 57 & & 0 \\
\hline LVX & & & 25 & 33 & & \\
\hline SXT & 10 & & & & 65 & 20 \\
\hline FOS & & & 0 & 0 & & \\
\hline NIT & & & 0 & 0 & 50 & 0 \\
\hline CLI & 18 & 26 & & & 28 & 29 \\
\hline OXA & 24 & 33 & & & 55 & 18 \\
\hline RIF & 0 & 0 & & & 20 & \\
\hline AZM & 0 & 0 & & & 33 & \\
\hline ERY & 28 & 47 & 39 & 33 & 70 & \\
\hline LNZ & 2 & 0 & 4 & 0 & 0 & 0 \\
\hline VAN & 0 & 0 & 4 & 0 & 0 & 0 \\
\hline TEH & 14 & 10 & 71 & 83 & 27 & 20 \\
\hline STH & & & 11 & 29 & & \\
\hline
\end{tabular}

AMP: Ampicilina. AMK: Amikacina. Ceftazidima. CLI Clindamicina. COL Colistin. CRO Ceftriaxona. CXM: Cefotaxima. CTX Cefotaxima. CZO Cefazolina. ERY: Eritromicina.ETP Ertapenem AZM Azitromicina. CIP: Ciprofloxacina.FOS Fosfomicina. FOX Cefoxitina. IPM: Imipenam. LNZ Linezolid.LVX Levofloxacina. MEM: Meropenem. NOR Norfloxacina. ATM: Aztreonam. GEN: Gentamicina. GEH: Gentamicina alta potencia. POL Polimixina. RIF Rifampicina. SAM: Ampicilina-Sulbactam. STH: Estreptomicina alta potencia. TEH Tetraciclina alta potencia. VAN: Vancomicina. TCC Ticarcilina-Ac. Clavulánico. TZP: Piperacilina-Tazobactam.. NET: Netilmicina. NIT Nitrofurantoína. SXT: Trimetoprim-Sulfametoxazol. PEN: Penicilina. OXA: Oxacilina.

Tabla 5. Resistencia bacteriana por bacterias de vigilancia y grupo antimicro-

$$
\text { biano GRAM POSITIVOS }
$$

Una resistencia de media a alta mostró staphylococcua aureus en relación a oxacilina $33 \%$, eritromicina $47 \%$, norfloxacina 67 \% y ampicilina $100 \%$. Una sensibilidad mayor a $74 \%$ se observó para clindamicina y otros antimicrobianos. No se observó resistencia para vancomicina y linezolid.

En el caso de enterococcus faecalis y faecium (3 cepas) mostraron alta resistencia para norfloxacina $57 \%$, eritromicina $39 \%$ y tetraciclinas $83 \%$. Una sensibilidad reducida se observó para levofloxacina $67 \%$, mientras que no se observó resistencia para ciprofloxacina, fosfomicina y nitrofurantoína.

Los aislados hospitalarios de staphylococus coagulasa negativa mostraron resistencia media a alta en relación a la mayoría de antimicrobianos probados con excepción de linezolid y vancomicina en todos los aislados COM y HOS. Los aislados COM de enterococcus mostraron resistencia los antibióticos probados de hasta un $20 \%$ siendo la más alta para clindamicina con $29 \%$.

\section{Evaluación de desempeño y calidad}

Siguiendo los lineamientos de evaluación calidad de la red de vigilancia de la OPS, se realizó la evaluación externa de desempeño con cepas ATCC que se entregaron codificadas como muestras A, B, C y D. Los resultados de desempeño se pueden ver en la Tabla 6. El laboratorio tuvo un $100 \%$ de concordancia en la identificación de género y especie de las cepas entregadas, sin embargo la cepa B no tuvo reporte de identificación con API, solamente registró la identificación con método manual y tipificación serológica.

En relación con el antibiograma reportado, de 39 resultados reportados, 92 \% estuvieron dentro del rango de control y 8 \% fuera de rango, sin embargo el $100 \%$ de los resultados fueron interpretados correctamente de acuerdo con la tabla y estándares CLSI 2010.

\section{Discusión}

Este es uno de los primeros estudios relacionados con resistencia bacteriana que relacionan los hábitos de prescripción empírica con la magnitud de la resistencia bacteriana en una comunidad atendida por un Hospital de tercer nivel. Hay evidencia que la incidencia de resistencia bacteriana está directamente relacionada con el uso de antibióticos, por tanto el conocimiento del uso de antibióticos y los patrones de resistencia a los mismos son necesarios para desarrollar guías de uso racional de antibióticos en la comunidad atendida por un hospital.

La vigilancia de la resistencia bacteriana en países en desarrollo es difícil y más cuando los antibióticos son de venta libre. Este estudio es un primer intento por identificar los hábitos de prescripción empírica de los médicos en el hospital y la comunidad 
y relacionarlos con los patrones de resistencia desarrollados por las bacterias aisladas de los pacientes atendidos en un área de salud de la Ciudad de Quito.

Aún cuando el método recomendado por la OMS para determinar el consumo de un medicamento es la Dosis Diaria Definida DDD/1000 pacientes ó DID, en éste estudio se analizó la frecuencia de prescripción empírica como una medida del uso de los diferentes antibióticos en las infecciones adquiridas en la comunidad y en el hospital. En los últimos años la tendencia a prescribir antibióticos de amplio espectro se ha duplicado y son los betalactámicos los más utilizados.(31)

Entre los factores que soportan la prescripción empírica predominan el resultado de antibiograma, la formación académica, la experiencia y el uso de guías internacionales. La encuesta aplicada permitió determinar que los antibióticos más prescritos empíricamente son los de amplio espectro, betalactámicos con o sin inhibidores de betalactamasas, cefalosporinas y fluoroquinolonas que se corresponden con las guías comerciales o de sociedades científicas utilizadas por nuestros médicos y que siguen la corriente mundial de prescripción.

La prevalencia de bacterias gram negativas y gram positivas aisladas en el laboratorio de muestras de origen comunitario y hospitalario tabla (2 y 3 ) mostraron que escherichia coli, staphylococcus aureus, pseudomona aeruginosa y otras enterobacterias, algunas de ellas de vigilancia recomendada por la OPS representan más del 83 \% de los aislamientos. Los patrones de resistencia mostrados se correlacionan con los antibióticos prescritos y con los patrones nacionales reportados por la Rednarbec en el 2009. Los problemas de resistencia en los países desarrollados están más asociados con gram positivos mientras que en los países en vías de desarrollo predominan los gram negativos. Es preocupante el porcentaje de enterobacterias productoras de betalactamasas, sin embargo el porcentaje de bacterias betalactamasas de espectro extendido BLEA se mantiene bajo. No se detectaron otras bacterias resistentes de vigilancia como MRSA, VRE, klebsiella pmeumoniae ó acinetobacter.

Los aislados urinarios fueron los más frecuentes, al igual que en otros estudios escherichia coli $82 \%$, otras enterobacterias $12 \%$ y enterococo 2 \% fueron los más aislados.

En cuanto a los aislados de piel, tejidos blandos y óseos predominan los aislados de escherichia coli 16 a $32 \%$, staphylococcus aureus 25 a 42 \%, pseudomona aeruginosa 6 a $10 \%$ en aislados HOS ó COM y otras enterobacterias 31 a 36 \% respectivamente.

Los aislados respiratorios predominan los gram negativos y staphylococcus aureus, no se aislaron streptococcus pneumoniae, haemophylus influenza ni moraxella catharralis que son los más frecuentemente reportados en otros estudios (21).

En las bacteriemias y sitios estériles también predominan escherichia coli y staphylococcus aureus y coagulasa negativos.

Los resultados del estudio se limitan a lo encontrado en un hospital público lo que no permite mantener una vigilancia sistemática sobre la resistencia bacteriana. La falta de colaboración de los médicos del Hospital para responder la encuesta de prescripción propuesta evidencia un sesgo en los resultados de prescripción empírica. Es importante analizar otros factores que influyen en la resistencia bacteriana y que se relacionan con la promoción farmaceútica, con los pacientes y el uso de antibióticos en la industria.

El presente estudio muestra una estrecha relación entre los hábitos empíricos de prescripción de los médicos institucionales y los patrones de resistencia desarrollados por las bacterias aisladas de muestras de la comunidad y el Hospital atendidos por esos médicos. Nuevos estudios deberán realizarse para establecer la relación entre el consumo de antibióticos y la resistencia bacteriana utilizando estándares internacionales recomendados por la OMS, sin 
embargo la fortaleza de este estudio es mostrar la magnitud actual de la resistencia bacteriana en una importante área de la comunidad atendida por un hospital de tercer nivel.

\section{Conclusión}

El uso de los antibióticos influye en la resistencia bacteriana entre otros factores. La vigilancia sistemática permite definir las tendencias de la resistencia bacteriana en un hospital y en la comunidad. Los médicos desconocen aspectos de vigilancia relacionados con la resistencia bacteriana y soportan su prescripción en instrumentos que se utilizan en otras realidades. Estos resultados sólo serán valiosos si sirven para implementar acciones de contención de la resistencia bacteriana como la elaboración de guías de uso racional de antibióticos ó políticas de restricción de uso de antibióticos en el hospital y en la comunidad, También deberián utilizarse para sugerir medidas de salud pública que contengan la emergencia de bacterias resistentes y el agravamiento del problema, caso contrario cada vez será más difícil tratar infecciones con los costos sociales y económicos que ello implica.

\section{Bibliografía}

Benavides-Plascencia Lilia, Leonardo Aldama-Ojeda Alejandro, Javier Vázquez Héctor. Vigilancia de los niveles de uso de antibióticos y perfiles de resistencia bacteriana en hospitales de tercer nivel de la Ciudad de México. Salud pública Méx .2005 June $47(3): 219-226$

Rosdahl, V. K, Pedersen. K. B. The Copenhagen Recommendations. Report from the Invitiational EU Conference on The Microbial Threat. Ministry of Health, Ministry of Food, Agriculture and Fisheries, Denmark. 2002:1-52 http://www.sum.dk/

David L. Smith*†, Anthony D. Harris*, Judith A. Johnson‡, Ellen K.
Silbergeld*, and J. Glenn Morris, Jr. Animal antibiotic use has an early but important impact on the emergence of antibiotic resistance in human commensal bacteria Biological Sciences. PNAS 200299 (9) 6434-6439; published ahead of print April 23, 2002, doi:10.1073/pnas.082188899

Ian Phillips1, Mark Casewell1, Tony Cox2, Brad De Groot3, Christian Friis4, Ron Jones5, Charles Nightingale6*, Rodney Preston7 and John Waddell8 Does the use of antibiotics in food animals pose a risk to human health? A critical review of published dataJ. Antimicrob. Chemother. (2004) 53 (1): 28-52. doi: 10.1093/jac/dkg483

World Health Organization. WHO Global Strategy for Containment of Antimicrobial Resistance. WHO/CDS/CSR/DRS/2001/2/EN. Disponible en: http://www.who.int/csr/resources/publication/ drugresist\%20/WHO_CDS_CSR_DRS_2001_2_EN/en/. [ Links ]

Center for Global Development. The race against drug resistance, A report of the Center for Global Development's Drug Resistance Working Group. Washington, DC: Center for Global Development; 2010

Carrera ME, Douce R, Zurita J. Costo de la neumonía nosocomial y bacteriana asociada a catéter venoso central en un hospital de Quito. En:Salvatierra-González,R ed. Costo de la infección nosocomial en nueve países de América Latina PAHO/OPS

Secretaría de Salud. Mexico DF.. Ley General de Salud, artículos 226,227.| fracción IV. www.salud.df.gob.mx/ssdf

Organización Panamericana de la Salud. Informe Anual de la Red de Monitoreo/Vigilancia de la Resistencia a los Antibióticos- 2009. Washington, D.C.

Zurita, J. y grupo REDNARBEC (2000) Vigilancia de la susceptibilidad antimicrobiana en Ecuador. En: Resistencia Antimicrobiana en las Américas: Magnitud del problema y su contención: 149-157. OPS/OMS, Washington, D.C. 
Life. Anexo 2

Helen W. Boucher1, George H. Talbot2, John S. Bradley3,4, John E. Edwards5,6,7, David Gilbert8, Louis B. Rice9,10, Michael Scheld11, Brad Spellberg5,6,7, and John Bartlett12.Bad Bugs, No Drugs: No ESKAPE! An Update from the Infectious Diseases Society of America.Clin Infect Dis. (2009) 48 (1): 1-12. doi: $10.1086 / 595011$

CLSI. Performance Standandars for Antimicrobial Susceptibility Testing; Twentieth Informational Supplement. USA 2010.

CLSI 2010 Normas para las pruebas de sensibilidad a los antimicrobianos con discos. Mo2-A10. Vol 29 No 1. Norma aprobada, décima edición. Enero 2010

Bosch FJ, van Vuuren C, Joubert G. Antimicrobial resistance patterns in outpatient urinary tract infections--the constant need to revise prescribing habits. S Afr Med J. 2011 May;101(5):328-31.

Center for Global Development. The race against drug resistance, A report of the Center for Global Development's Drug Resistance Working Group. Washington, DC: 2010

Helen W. Boucher1, George H. Talbot2, John S. Bradley3,4, John E. Edwards5,6,7, David Gilbert8, Louis B. Rice9,10, Michael Scheld11, Brad Spellberg5,6,7, and John Bartlett12.Bad Bugs, No Drugs: No ESKAPE! An Update from the Infectious Diseases Society of America.Clin Infect Dis. (2009) 48 (1): 1-12. doi: $10.1086 / 595011$

Anatomical Therapeutical Chemical Classification Index. Including defined daily dosis (DDD) for plain substances. Oslo: WHO Collaborating Centre for Drug Statistics Methodology, 1995

Kärpänoja P, Nyberg ST, Bergman M, Voipio T, Paakkari P, Huovinen P, Sarkkinen H, Finnish Study Group for Antimicrobial Resistance (FiRe Network). Connection between trimethoprimsulfamethoxazole use and resistance in Streptococcus pneumoniae, Haemophilus influenzae, and Moraxella catarrhalis. Antimicrob Agents Chemother. 2008 Jul; 52(7):2480-5. Epub 2008 Apr 28.

Butler CC, Dunstan F, Heginbothom M, Mason B, Roberts Z, Hillier S, Howe R, Palmer S, Howard A. Containing antibiotic resistance: decreased antibiotic-resistant coliform urinary tract infections with reduction in antibiotic prescribing by general practices. Br J Gen Pract. 2007 Oct; 57(543):785-92.

Sow Al, Boye A, Ka Sall R, Ndour CT, Soumare M, Seydi M, Samb A [Resistance of bacteria and antibiotic prescription in Fann University Teaching Hospital, Dakar]. Dakar Med. 2003;48(3):189-93. PMID:15776629

Romo H. Uso de antibióticos. En prensa.

Porretta A, Giuliani L, Vegni FE, Larosa M, Privitera G; INF-NOS Study Group. Prevalence and patterns of antibiotic prescribing in Italian hospitals. Infection. 2003 Dec;31 Suppl 2:16-21.

Bergman M, Huikko S, Huovinen P, Paakkari P, SeppÃalÃa H; Finnish Study Group for Antimicrobial Resistance (FiRe Network). Macrolide and azithromycin use are linked to increased macrolide resistance in Streptococcus pneumoniae. Antimicrob Agents Chemother. 2006 Nov;50(11):3646-50. Epub 2006 Aug 28.

Arnold SR, Straus SE. Interventions to improve antibiotic prescribing practices in ambulatory care.Cochrane Database Syst Rev. 2005 Oct 19;(4)

Fishman N. Antimicrobial stewardship. Am J Infect Control. 2006 Jun;34(5 Suppl 1):S55-63; discussion S64-73 (a)

Gales AC, Sader HS, Jones RN; SENTRY Participants Group (Latin America).Urinary tract infection trends in Latin American hospitals: report from the SENTRY antimicrobial surveillance program (1997-2000). Diagn Microbiol Infect Dis. 2002 Nov;44(3):289-99 
Boucher HW, Talbot GH, Bradley JS, Edwards JE, Gilbert D, Rice LB, Scheld M, Spellberg B, Bartlett J. Bad bugs, no drugs: no ESKAPE! An update from the Infectious Diseases Society of America. Clin Infect Dis. 2009 Jan 1;48(1):1-12.

De Souza V, MacFarlane A, Murphy AW, Hanahoe B, Barber A, Cormican M.A qualitative study of factors influencing antimicrobial prescribing by non-consultant hospital doctors. J Antimicrob Chemother. 2006 Oct;58(4):840-3. Epub 2006 Aug 2

Kotwani A, Wattal C, Katewa S, Joshi PC, Holloway K. Factors influencing primary care physicians to prescribe antibiotics in Delhi India. Fam Pract. 2010 Dec;27(6):684-90. Epub 2010 Jul 26 